\title{
Five Years Follow-Up of Adjustable Continence Therapy (ACT) in the Treatment of Recurrent Female SUI ${ }^{*}$
}

\author{
Niall T. Galloway ${ }^{1}$, Sherif R. Aboseif ${ }^{2}$, Pejvak Sassani ${ }^{2}$, Ethan I. Franke ${ }^{2}$, Steven D. Nash ${ }^{3}$, \\ Joel N. Slutsky ${ }^{4}$, Mai Le Tu ${ }^{5}$, Peter J. Pommerville ${ }^{6}$, Neil H. Baum ${ }^{7}$, Suzette E. Sutherland ${ }^{8}$ \\ ${ }^{1}$ Emory University School of Medicine, Atlanta, USA \\ ${ }^{2}$ Kaiser Permanente Medical Center, Los Angeles, USA \\ ${ }^{3}$ Kansas City Urology Care, Leawood, USA \\ ${ }^{4}$ Urological Surgeons, Kankakee, USA \\ ${ }^{5}$ CHUS Flairmont, Quebec City, Canada \\ ${ }^{6}$ Can-Med Clinical Research Inc., Victoria BC, Canada \\ ${ }^{7}$ Neil Baum Urology, New Orleans, USA \\ ${ }^{8}$ Metro Urology, Centers for Continence Care and Female Urology, Minneapolis/St. Paul, USA \\ Email: adam.shrewsberry@gmail.com
}

Received December 28, 2012; revised January 31, 2013; accepted February 9, 2013

Copyright (C) 2013 Niall T. Galloway et al. This is an open access article distributed under the Creative Commons Attribution License, which permits unrestricted use, distribution, and reproduction in any medium, provided the original work is properly cited.

\begin{abstract}
Purpose/Objective: The primary objective of this FDA study is to evaluate the safety and efficacy of the ACT device (Adjustable Continence Therapy) in the treatment of female recurrent Stress Urinary Incontinence (SUI). The secondary objective is to evaluate the difficulty of placement and aspects of device adjustability. Materials and Methods: The Adjustable Continence Therapy (ACT) system (Uromedica, Inc., Plymouth, MN) consists of two silicone balloons providing urethral coaption and bladder neck support. Each balloon is attached to a titanium port buried in the labia allowing for post-operative adjustment of volume. Females with recurrent SUI with or without urethral hypermobility were evaluated at baseline and follow-up periods of 6 weeks, 3, 6, 9, 12 months and annually thereafter including urinalysis, a 3-day voiding diary, provocative pad weight test, direct visual stress test, Stamey score and validated questionnaires to assess severity of incontinence, voiding dysfunction, sexual function and quality of life. Results: During a 5 years period (2002-2007), 162 patients were implanted (mean age 67.6, range 31 - 94 years). Of these 162 patients 142, 90, 80, 56 and 31 patients completed the 1, 2, 3, 4 and 5 years follow-up respectively. One hundred and thirty five (83\%) had failed at least one surgery for incontinence and $44 \%$ had failed 2 or more procedures prior to ACT implantation. Difficulty of ACT placement was rated mild in $62 \%$, moderate in $30 \%$ and severe in $9 \%$. Improvement in Stamey score of $>1$ grade was achieved in $75 \%(107 / 142)$ at 1 year, $76 \%(68 / 90)$ at 2 years, $86 \%(62 / 78)$ at 3 years, 93\% (50/54) at 4 years and $83 \%(25 / 30)$ at 5 years. Dry rate (provocative pad weight $<2$ gms) was $51 \%, 62 \%, 76 \%, 76 \%$ and $76 \%$ at 1 through 5 years, and $>50 \%$ improvement was achieved in $83 \%, 86 \%, 86 \%, 90 \%$ and $93 \%$,respectively. IQoL improved from 37 at baseline to 71, 71, 75, 77 and 74 during the study, and optimal continence was achieved with a mean of 4 adjustments, and mean balloon volumes of $4.0 \mathrm{ml}(1.0-11.5 \mathrm{ml})$. Complications including bladder perforation, erosion, migration, pain and urinary retention were reported in 25\% (38/155) at 12 months, and 9\% (10/109), 3\% (3/91), 6\% $(5 / 79)$ and $4 \%$ (3/69) respectively, and of these, the majority were mild to moderate. At 5 years, 33 patients had undergone permanent explants of both devices, 48 were lost to follow-up and 6 had died of unrelated causes. Conclusions: Five years data suggest that ACT is, a safe and effective, minimally invasive treatment for recurrent SUI, which is easy to place and adjust to optimize urinary control without impairing bladder emptying.
\end{abstract}

Keywords: Female Urinary Incontinence; Implant; Balloon Adjustments; Safety; Stamey Score

\section{Introduction}

Stress urinary incontinence (SUI) is a major urologic

${ }^{*}$ Conflict of interest: The authors declare that they have no conflict of interest. health issue that affects millions of patients each year. SUI is characterized by the loss of urine with exertion coughing, sneezing or lifting [1] SUI can result from hypermobility of the bladder neck and urethra associated with defects of pelvic support anatomy or from intrinsic 
sphincter deficiency (ISD). ISD is due to lack of coaption of the urethral wall and is diagnosed when a well-supported urethra leaks urine in response to a slight increase in intra-abdominal pressure in the absence of detrusor contraction.

Multiple surgical procedures including retro pubic suspension, and mid-urethral sling are effective in primary cases, but recurrent SUI secondary to ISD continues to be a clinical challenge, awaiting an ideal treatment option [2]

Standard surgical treatments for ISD include slings, artificial urinary sphincter, use of periurethral bulking agents or urethral remodeling by radiofrequency (RF). Effective treatments for ISD increase urethral resistance [3] and correcting urethral hypermobility alone may not improve symptoms of stress urinary incontinence [4]. The introduction of adjustable periurethral devices presents an opportunity to balance increased urethral resistance and effective bladder emptying [5]. The implantable ACT device can be titrated with time as required and offers an alternative surgical option in women with recurrent stress urinary incontinence. We have evaluated the safety and efficacy of the ACT device and also assessed the difficulty of placement and device adjustability over a five years period.

\section{Materials and Methods}

\subsection{The ACT Device}

ACT consists of two silicone elastomer fluid filled balloons placed one on each side of the proximal urethra, and connected via a conduit to a titanium port buried superficially in the fatty tissue of the labia majora [6,7] The balloons are placed using two specially designed reusable trocars (sharp and blunt tipped for tissue dissection) and a U-shaped cannula. The device is available in 2 lengths $(8$ and $9 \mathrm{~cm})$ and the most suitable device length for each patient is determined by urethral measurement using the trocar and cannula. At any time post operatively (years later if need be) the volume in each balloon can be adjusted by percutaneous injection or aspiration of fluid through the port using a 23 gauge Huber non coring needle to achieve optimum continence with a recommended maximum volume of $8 \mathrm{cc}$. The possibility of percutaneous adjustment of the sizes of the balloons according to clinical need to achieve clinical efficacy appears to be an essential advantage of this technique [8].

\subsection{Study Design}

This was an FDA investigational open label study. Upon receiving ethical approval we performed a prospective, non-randomized open study to access the potential of this adjustable device in women to treat SUI. ACT is a novel design, with no predicate and hence was unable to blind the patient. Selection criteria included adult female patients with symptomatic SUI with urethral hypermobility and/or ISD and who had failed previous treatment over at least 6 months. The exclusion criteria included a diagnosis of_neurogenic bladder/detrusor dysfunction, prior pelvic radiation therapy, insulin dependent diabetes, and uncorrected moderate or severe pelvic organ prolapse.

Physical examination, included assessment of urethral mobility and urodynamics included measurement of baseline Valsalva leak point pressure (VLPP) and maximum urethral closure pressure (MUCP). Outcomes were evaluated using Stamey Score, provocative pad weight test, daily pad count, incontinence quality of life questionnaire (IQoL) and the visual analog scale measured at baseline, 6 weeks, 3 months, 6 months, 9 months, 12 months and annually. Patients also completed the IIQ/ PiSQ and IQoL scoring scheme at each post-operative visit.

The surgical procedure was done as previously described [6]. The ACT devices were placed bilaterally using small incisions in the labial folds at the level of the urethral meatus. A specially designed delivery trocar is passed under the fluoroscopic and digital vaginal guidance through each incision and positioned just distal to the bladder neck. After placement of each device the balloons are inflated with $1.5 \mathrm{ml}$ of an isotonic contrast solution and fluoroscopy, cystoscopy and vaginal palpation were used to confirm proper positioning of the balloons. The first balloon adjustment is done 6 weeks post operatively and then adjusted every 4 weeks afterwards (maximum of $1 \mathrm{~mL}$ per balloon per adjustment) until adequate continence is achieved by subjective and objective criteria.

\subsection{Statistical Analyses of Study Outcome}

Statistical analyses were completed using the non-parametric Wilcoxon's matched pairs signed ranks test to compare baseline and follow-up values to determine efficacy outcomes, and $p$ values of $<0.05$ were regarded as statistically significant. The data are presented in both "As followed" and "Intent to Treat" analyses. The "As followed" (AF) analyses included only those who complied with the protocol and interval follow-up, the "Intent to Treat" (ITT) included all participants whether active, non-active, withdrawn, died, explanted or lost to followup. If a subject failed to reach the 12-month end point they were considered as failures and the baseline value was used at the 12-month end point.

The Stamey score was required as the FDA primary efficacy clinical end point which represented a reduction of at least 1grade from the baseline at 12 months after implantation, additional measures included direct visual 
stress test, 3 days voiding diary (number of leaks and number of pads/day), provocative 1 hour pad weight test and validated questionnaires (IIQ/PiSQ and IQoL). An independent reviewer scored Stamey grades to validate the primary endpoint and assess poolability of results between study sites.

Safety was studied using all adverse events related to the device or procedure, and technical feasibility was assessed and recorded by the surgeons at the time of implantation and at interval volume adjustments.

All analyses were performed using the statistical software version of the Statistical Package for Social Sciences (SPSS).

\section{Results}

A total of 162 subjects (mean age 67.6 years, range 31 94) were implanted. Of these $83 \%(134 / 162)$ had failed one or more prior surgeries, including slings, suspensions and bulking agents. The distribution of patients who failed prior procedures are $38 \%, 32 \%, 8 \%$ and $4 \%$ for 1 , 2,3 and 4 or more procedures respectively. There was $88 \%$ subject visit compliance at 12 months (142/162), $12 \%$ did not comply ( 4 missed follow-up, 7 were permanently explanted, 8 were lost to follow-up and there was 1 death).

The patient visit compliance from implantation to the 5 years follow-up period was 99\%, 87.6\%, 55.6\%, 49.4\%, $34.6 \%, 19.1 \%$ from implantation, $1,2,3,4$ and 5 years respectively (Table $\mathbf{1}$ ).

The data demonstrates that $67 \%, \mathrm{n}=162$ (ITT) and $75.4 \%, \mathrm{n}=142$ (AF) showed an improvement in Stamey score and the sensitivity analyses demonstrated consistency of observed treatment effects by statistical methods used. The improvement of Stamey score over the 5 years follow-up period is shown in Figure 1. Primary efficacy durability (Figure 2) shows a mean reduction 1.32 at 12 months and 1.37 at 5 years.

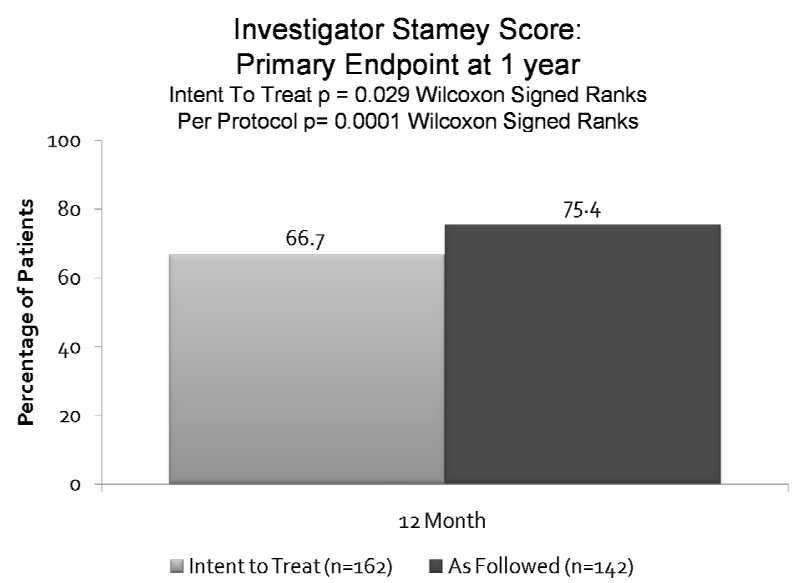

Figure 1. Investigator Stamey score Primary Endpoint at 1 year.
The mean one hour provocative pad weight at pre-implant was $54 \mathrm{~g}$ for both IIT and AF analyses and at 12 months they were $22.7 \mathrm{~g}$ and $11.1 \mathrm{~g}$, (p value respectively (Figure 3)). At 12 months 64.2\% (ITT) and 79.7\% (AF) patients demonstrated pad weight reduction of $>50 \%$ (Figure 4). Quality of life showed a marked improvement from 36.8 at baseline to 74.3 at 5-year period (Figure 5).

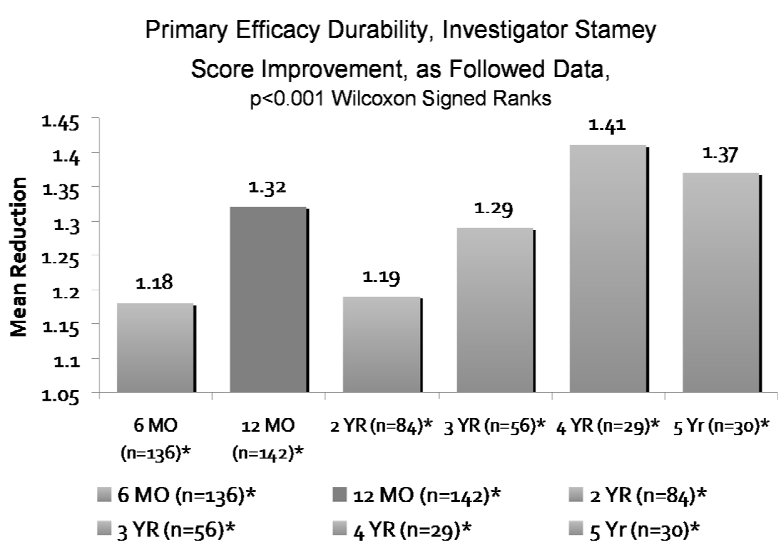

Figure 2. Primary efficacy durability.

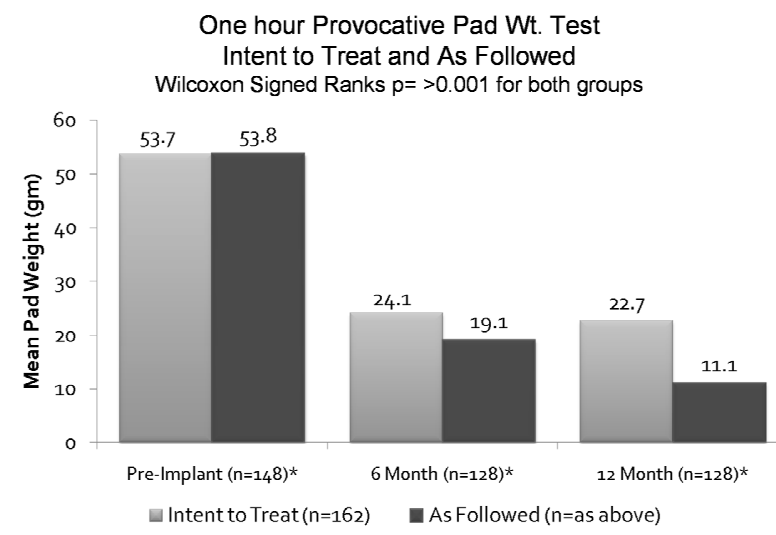

Figure 3. One hour provocative pad wt IIT vs AF.

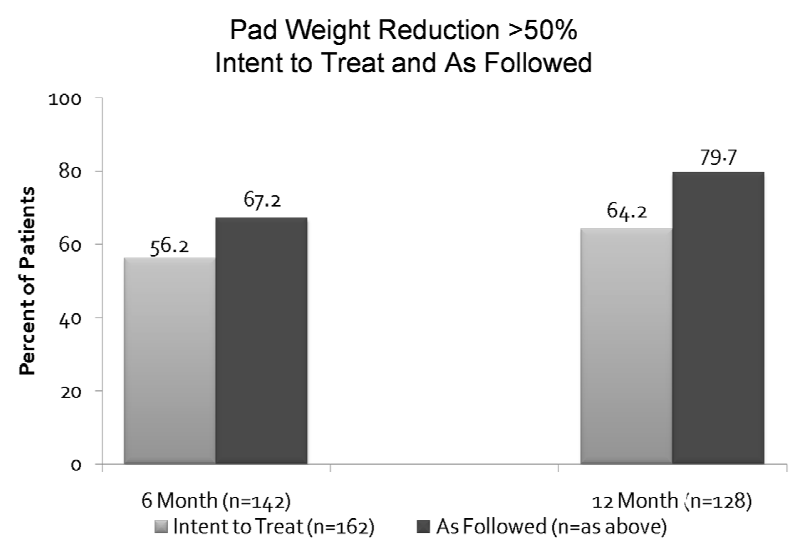

Figure 4. Pad weight reduction of $50 \%$ or more of ITT vs AF. 
Table 1. Subject visit compliance by follow-up in years.

Study subjects: Visit compliance

\begin{tabular}{|c|c|c|c|c|c|c|}
\hline Study visit & Implant & 1 Year & 2 Years & 3 Years & 4 Years & 5 Years \\
\hline Complete F/up & 161 & 142 & 90 & 80 & 56 & 31 \\
\hline Perm. Explanted & 1 & 7 & 18 & 24 & 30 & 33 \\
\hline Lost to follow-up & 0 & 8 & 30 & 36 & 44 & 48 \\
\hline Death & 0 & 1 & 5 & 6 & 6 & 6 \\
\hline MD Withdrew pt & 0 & 0 & 0 & 0 & 0 & 1 \\
\hline Withdrew to $\mathrm{AE}$ & 0 & 0 & 0 & 0 & 0 & 1 \\
\hline Completed study & 0 & 0 & 5 & 5 & 5 & 5 \\
\hline No eligible F/up & 0 & 0 & 0 & 0 & 6 & 25 \\
\hline Total & 162 & 162 & 162 & 162 & 162 & 162 \\
\hline
\end{tabular}

Quality of Life and Distress Measures Improved

\begin{tabular}{|l|l|l|l|l|l|l|}
\hline & Baseline & $\mathbf{1 ~} \mathbf{~ r ~}$ & $\mathbf{2} \mathbf{~ r ~}$ & $\mathbf{3} \mathbf{Y r}$ & $\mathbf{4} \mathbf{~ r r}$ & $\mathbf{5} \mathbf{~ r r}$ \\
\hline UDI & 60 & 37 & 44 & 44 & 48 & 51 \\
\hline IQoL & 36.8 & 71.1 & 70.9 & 74.8 & 77.6 & 74.3 \\
\hline
\end{tabular}

- Surgical Technique

- Degree of Difficulty

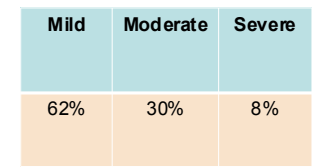

Figure 5. Quality of life scores over follow-up period.

There were no serious complications reported and no serious risks associated with the device.

The mean number of balloon adjustments dropped from 2.1 at 12 months to 0.17 at 4 to 5 years, indicating that balloons were well placed and continence was achieved and did not need major adjustments (Figure 6).

\section{Discussion}

Stress urinary incontinence affects a large proportion of women and has a negative impact on quality of life. Surgical procedures such as mid urethral slings offer high success that have made them attractive choices for primary cases [9], and the complication profile includes bladder, bowel and blood vessel injuries, post-operative voiding difficulties, de novo urgency and urge incontinence [10]. Patients who have had prior incontinence procedures have less favorable outcomes, Houwert [11] reports $82 \%$ of patients who have not had prior surgery were improved after mid-urethral sling while that percentage was only $56 \%$ for patients who have had prior

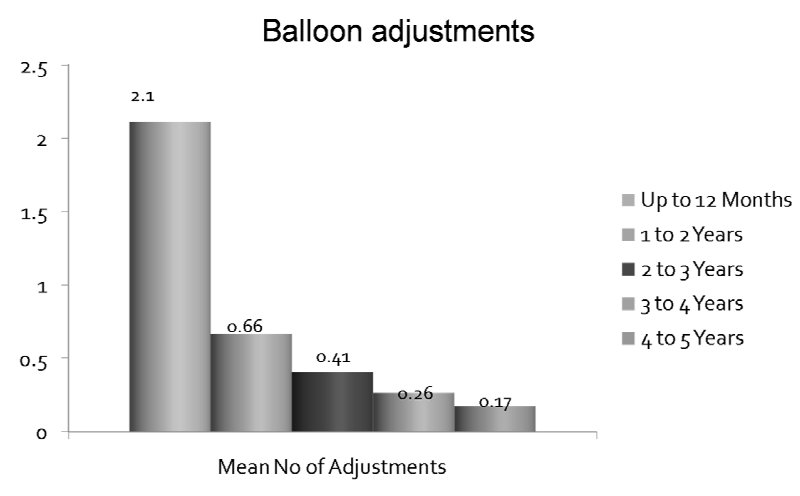

Figure 6. Mean number of balloon adjustments over followup period.

incontinence surgery. It appears that the proper adjustment of sling tension can be difficult in recurrent cases of stress incontinence and a challenge even in the most experienced hands. The efficacy of sling procedures is correlated to severity of SUI. An article by Song et al. on a 7-year outcomes of TVT suggests that patients with high grade SUI (Stamey grade III) have a lower cure rate $(50 \%)$, while patients with moderate (Stamey grade II) SUI have a cure rate of $82.8 \%$, and patients with grade I have a cure rate of $90.7 \%$. [12]

Procedures using injectable periurethral bulking agents, or urethral sub mucosal collagen, denaturation by radiofrequency (RF) [6] are less invasive, but like the mid urethral sling offer no possibility of adjustment to balance bladder control with bladder emptying. Another exciting treatment option is the introduction of stem cells in an effort to regenerate the sphincter muscle and improve function [13]. Mittenberger et al. has suggested promising results for the treatment of SUI, but other 
groups have failed to duplicate their results [13].

Many studies have been published on the clinical utility of the adjustable continence therapy device for treatment of stress urinary incontinence in both Europe and North America.

A study by O'Connell H.E. et al. [14] on the extent of structural disruptions caused by surgical insertion of the ACT device and possible impact of this device on female sexual anatomy concluded that there is no significant disruption and that it was anatomically safe with respect to perineal anatomical structures.

Magnetic Resonance Imaging (MRI) was used to understand how the ACT implantation provides continence, and as a tool for radiological assessment of continence recovery by Stecco et al. [15]. It was concluded that MRI provided an effective radiological method to predict the efficacy of ACT. Chartier-Kastler et al. [16] reported $87 \%$ improvement, and Kocjancic et al. [17,18] reported $68 \%$ dry and $16 \%$ improved and Aboseif et al. showed that $76.4 \%$ of patients with either, moderate or severe stress incontinence showed at least one grade improvement of the Stamey score and the mean provocative pad weight decreased by more than $50 \%$ in $80.9 \%$ of the patients [19] Aboseif et al. reported that $47 \%$ of patients were dry at 1 year and 92\% improved after 1-year follow-up [20]. The ACT device can be implanted in patients presenting with urinary incontinence due to sphincter deficiency with no urethral hypermobility (negative support maneuvers), in cases of failures with other therapies, or when placing an AUS is contraindicated or refused by the patient. [21,22]. Vayleux et al. [23] observed in their study that ACT is an attractive alternative owing to its benefit-risk ratio in which the technique of using a flexible cystoscopy and bladder neck "retrovision" would improve the clinical result for safety and efficacy of ACT surgery by more precisely placing the balloons at the bladder neck. .

The scope of this study is to evaluate the safety, efficacy and technical feasibility the Adjustable Continence Therapy (ACT) device (Uromedica Inc. Plymouth MN, USA) over a five years follow-up period. There is less opportunity to introduce investigator bias in the "Intentto-Treat" methodology and this was used to provide a more conservative measure of efficacy than the "As followed" analyses which are also included. As follow-up is extended to beyond 5 years, there is survivor bias that is unavoidable, because data is available on that cohort of subjects who continue to be followed and not in all of the initial cohort implanted. It is unusual to observe improved continence over time as was observed in this study, and this reflects the adjustable nature of the ACT therapy which can be optimized by adding more isotonic fluid volume into the balloons, in small increments using simple injection via the titanium port sited subcutane- ously in the labial fold.

We observed an improvement in Stamey score of $>1$ in $75.4 \%$ at 1 year and $83.3 \%$ at 5 years. The dry rate (PPWT $<2$ gms) was $51 \%$ at 1 year and improved to $76 \%$ at 5 years and $>50 \%$ improved rate was $83 \%$ at 1 year and $93.1 \%$ at 5 years. IQoL scores showed an increase from 36.8 at baseline to 74.3 at 5 years, suggesting that the ACT device significantly improves quality of life for the patient and that it is sustained over time.

The salient feature of the device is the ease of adjustability [24], adding volume by increments to balance bladder control with voiding function to suit the needs of the individual patient in a simple procedure that is done in the clinic exam room. Optimal continence was achieved with a mean of 4 adjustments and mean balloon volume of $4.0 \mathrm{ml}(1-11.5 \mathrm{ml})$.

Device or procedure related complications (bladder perforation, port or balloon erosion, balloon migration, port or balloon related discomfort, intermittent urinary retention) was reported in $25 \%$ at 12 months and only $4 \%$ in 5 years. No major complications are reported and in a multi-center multiple surgeon study with a novel surgical device and technique, some of the complications reported may be explained by initial learning curve of surgical experience. The complications that did occur were minor and resolvable, and when required easily removable in the clinic setting, indicating that $\mathrm{ACT}$ is safe to use in clinical practice.

\section{Conclusion}

Our five years follow-up data suggest ACT is simple, safe, minimally invasive an effective alternative treatment for mild, moderate or severe recurrent female SUI.

\section{REFERENCES}

[1] P. Abrams, L. Cardozo, M. Fall, et al., "The Standardization of Terminology of the Lower Urinary Tract Function: Report from the Standardization Sub-Committee of the International Continence Society," Neurourology and Urodynamics, Vol. 21, No. 2, 2002, pp. 167-178. doi:10.1002/nau.10052

[2] P. Sassani and S. R. Aboseif, "Stress Urinary Incontinence in Women," Current Urology Reports, Vol. 10, No. 5, 2009, pp. 333-337. doi:10.1007/s11934-009-0052-5

[3] E. Kocjancic, S. Crivellaro, S. Ranzoni, D. Bonvini, B. Grosseti and B. Frea, "Adjustable Continence Therapy for Severe Intrinsic Sphincter Deficiency and recurrent Female Stress Urinary Incontinence: Long-Term Experience," The Journal of Urology, Vol. 184, No. 3, 2010, pp. 1017-1021. doi:10.1016/j.juro.2010.05.024

[4] C. Pope, P. J. R. Shaw and M. J. Coptcoat, "Changes in Bladder Function Following a Surgical Alteration in Outflow Resistance," Neurourology and Urodynamics, Vol. 18, 1999, p. 629. 
[5] S. Crivellaro and J. J. Smith, "Minimally Invasive Therapies for Female Stress urinary Incontinence: Current Status of Bioinjectables/New Devices (Adjustable Continence Therapy, Urethral Sub Mucosal Collagen Denaturation by Radiofrequency)," The Scientific World Journal, Vol. 9, 2009.

[6] J. Wachter, A. Henning, M. Roehlich, et al., "Adjustable Continence Therapy for Female Urinary Incontinence: A Minimally Invasive Option for the Difficult Case," International Journal of Urology, Vol. 81, 2008, pp. 160-166. doi:10.1159/000144054

[7] L. Le Normand, "Les Ballonets Ajustables (ACT®) Periuretraux Pour le Traitement de l'Incontinencede la Femme," Pelvi-Perineal, Vol. 1, 2007, pp. 1-5.

[8] E. Chartier-Kastler, P. Costa, K. B. Naoum, F. Cour, L. Le Normand and F. Haab, "Prospective Multicenter French Study on the Use of ACT ${ }^{\circledR}$ Balloons for the Treatment of Urinary Incontinence in Women," Progrès en Urologie, Vol. 17, No. 7, 2007, pp. 1372-1377. doi:10.1016/S1166-7087(07)78580-9

[9] S. Kuschel and B. Schussler, "Results of Function and Safety of the Safyre-t, a Hybrid Transobturator Vaginal Sling for the Treatment of Stress Urinary Incontinence," Neurourology and Urodynamics, Vol. 27, No. 5, 2008, pp. 403-406. doi:10.1002/nau.20528

[10] E. David-Montefiore, J. L. Frobert, M. Grisard-Anaf, et al., "Functional Results after Sub Urethral Sling Procedure for Urinary Stress Incontinence at 1 Year: A French Prospective Multicenter Study Comparing the Retropubic and Transobturator Routs," International Urogynecology Journal and Pelvic Floor Dysfunction, Vol. 17, Suppl. 2, 2006, p. S95.

[11] R. M. Houwert, P. L. venema, A. E. Aquarius, et al., "Predictive Value of Urodynamics on the Outcome after Midurethral Sling Surgery for Female Stress Urinary Incontinence," American Journal of Obstetrics and Gynecology, 2009. www.AJOG.org

[12] P. H. Song, Y. D. Kim, H. D. Kim, et al., "The 7-Year Outcome of the Tension Free Vaginal Tape Procedure for Treating Female Stress Urinary Incontinence," BJU International, Vol. 104, No. 8, 2009, pp. 1113-1117. doi:10.1111/j.1464-410X.2009.08504.x

[13] M. Mittenberger, G. M. Pinggera, R. Marksteiner, et al., "Adult Stem Cell Therapy for Female Stress Urinary Incontinence," European Urology, Vol. 53, No. 1, 2008, pp. 169-175. doi:10.1016/j.eururo.2007.07.026

[14] K. E. O'Connell, S. Kalavampara, B. Frea, P. Robertson and E. Kocjancic, "Cadaveric Study of ACT Balloons and Their Impact on Female Sexual Anatomy," Pelviperineology, Vol. 26, 2007, pp. 53-56.

[15] A. Stecco, A. Saponaro, S. Crivellaro, C. Raffaele, A. Otroneo, B. Frea, A. Carriero and E. Kocjancic, "Can MRI Predict Which Patients Are Most Likely to Benefit from Percutaneous Positioning of Volume-Adjustable
Balloon Devices?" Urologia Internationalis, Vol. 76, 2006, pp. 240-246. doi:10.1159/000091627

[16] E. Chartier-Kastler, P. Costa, K. Ben, Naoum, et al., "French Multicenter Prospective Study of the Use of ACT Balloons for the Treatment of Female Stress Urinary Incontinence," Progrès en Urologie, Vol. 17, No. 7, 2007, pp. 1372-1377. doi:10.1016/S1166-7087(07)78580-9

[17] E. Kocjanacic, B. Frea, P. Robertson and H. E. O'Connell, "Anatomical Basis for Effective Placement of Adjustable Continence Therapy (ACT) Balloons for Treatment of Female Stress Urinary Incontinence," Pelviperineology, Vol. 26, 2007, pp. 88-90.

[18] E. Kocjancic, S. Crivellaro, J. J. Smith, et al., "Adjustable Continence Therapy for Treatment of Recurrent Female Urinary Incondinence," Journal of Endourology, Vol. 22, No. 7, 2008, pp. 1403-1407. doi:10.1089/end.2008.0027

[19] S. R. Aboseif, E. I. Franke, S. D. Nash, J. N. Slutsky, N. H. Baum, M. Le Tu, N. T. Galloway, P. J. Pommerville, S. E. Sutherland and J. F. Bressette, "The Adjustable Continence Therapy (ACT) System for Recurrent Female Stress Urinary Incontinence; One Year Results of the North America Clinical Study Group," Journal of Urology, Vol. 181, No. 5, 2009, pp. 2187-2191. doi:10.1016/j.juro.2009.01.039

[20] S. R. Aboseif, P. Sassani, E. I. Franke, S. D. Nash, J. N. Slutsky, N. H. Baum, M. Le Tu, N. T. Galloway, P. J. Pommerville and S. E. Sutherland, "Treatment of Moderate to Severe Female Stress Urinary Incontinence with the Adjustable Continence Therapy (ACT) Device after Failed Surgical Repair," WJU, Vol. 29, No. 2, 2010, pp. 249-253.

[21] A. Vidart and F. Cour, "Guidelines for the Treatment of Non-Neurological Urinary Incontinence Women Using Periurethral Balloons," Progrès en Urologie, Vol. 20, Suppl. 2, 2010, pp. S150-S154. doi:10.1016/S1166-7087(10)70010-5

[22] B. Vayleux, F. Luyckx, S. Thelu, J. Rigaud, O. Bouchot, G. Karam, L. Le Normand, "Resultats Fonctionnels des Ballonnets Periuretraux ACT Chez la Femme (Functional Results of Adjustable Continence Therapy ${ }^{\circledR}$ in Women), Pelvi-Perineologie,Vol. 5, No. 2, 2010, pp. 83-89. doi:10.1007/s11608-010-0304-3

[23] B. Vayleux, F. Luyckx, S. Thelu, J. Rigaud, O. Bouchot, G. Karam, L. Le Normand, "Les Ballonets Périurétraux ACT ${ }^{\circledR}$ Chez la Femme: Suivi à Moyen Terme et Aide au Positionnement," Progrès en Urologie, Vol. 20, No. 7, 2010, pp. 420-526. doi:10.1016/j.purol.2010.01.010

[24] E. Chartier-Kastler, P. Costa, K. B. Naoum, F. Cour, L. Le Normand and F. Haab, "Prospective Multicenter French Study on the Use of ACT ${ }^{\circledR}$ Balloons for the Treatment of Urinary Incontinence in Women," Progrès en Urologie, Vol. 17, No. 7, 2007, pp. 1372-1377. doi:10.1016/S1166-7087(07)78580-9 